\title{
The Safety and Efficacy of Repetitive Transcranial Magnetic Stimulation in the Treatment of Major Depression Among Children and Adolescents: A Systematic Review
}

\author{
Pradipta Majumder ${ }^{1}$, Sabish Balan ${ }^{2}$, Vikas Gupta ${ }^{3}$, Roopma Wadhwa ${ }^{4}$, Tarique D. Perera ${ }^{5}$ \\ 1. Psychiatry, WellSpan, York, USA 2. Psychiatry, Harlem Hospital, New York, USA 3. Psychiatry, South Carolina \\ Department of Mental Health, Columbia, USA 4. Psychiatry and Behavioral Sciences, South Carolina Department of \\ Mental Health, South Carolina, USA 5. Psychiatry and Behavioral Sciences, Contemporary Care, Greenwich, USA
}

Corresponding author: Pradipta Majumder, drpradipta@yahoo.co.in

\begin{abstract}
Major depression is a chronic debilitating condition affecting people of all ages and is rising over the past decade. Major depression among children and adolescents is often resistant to traditional treatments, thus necessitating the exploration of novel strategies. Repetitive transcranial magnetic stimulation (rTMS) is gaining increasing attention as a useful tool in treating various conditions and has received the US Food and Drug Administration (FDA) approval to treat depression and obsessive-compulsive disorder among adults. Favorable outcomes among adults generated interest in using it among children. Until recently, the existing literature lacked randomized sham-controlled trials on this topic among children and adolescents. The newest additions in the literature necessitated another in-depth look at the data to explore the safety and efficacy of rTMS in the context of depression among children and adolescents. We searched the Medline and Cochrane databases and included 18 articles for our systematic review. Our systematic review indicates level 1 evidence that rTMS is safe but failed to show its superiority to placebo as a stand-alone treatment for resistant depression among children and adolescents. However, there is level 2 evidence favoring add-on rTMS to treat major depression among children and adolescents. The study subjects appear to tolerate the rTMS treatment well with some minor and mostly self-limited side effects. Risks of treatment-emergent hypomanic symptoms and seizure appear to be very low. There is no evidence of worsening of suicidal ideation or cognitive decline during rTMS treatment.
\end{abstract}

Review began 04/06/2021 Review ended 04/13/2021 Published 04/19/2021

\section{(c) Copyright 2021}

Majumder et al. This is an open access article distributed under the terms of the Creative Commons Attribution License CC-BY 4.0., which permits unrestricted use, distribution, and reproduction in any medium, provided the original author and source are credited.
Categories: Psychiatry

Keywords: transcranial magnetic stimulation, adolescent, pediatric, depression, randomized control trial

\section{Introduction And Background}

The rate of major depression has increased from $8.7 \%$ in 2005 to $11.3 \%$ in 2014 in adolescents [1]. Major depression in childhood is often associated with school dropout, unemployment, and unwanted pregnancy [2]. The Treatment for Adolescents with Depression Study showed that a combination of fluoxetine and cognitive-behavioral therapy was associated with a higher remission rate in adolescent depression than either treatment alone [3]. Nevertheless, almost 30-40\% of depressed adolescents fail to respond to traditional treatment $[4,5]$. There are also concerns about treatment-emergent side effects, such as suicidality with the use of antidepressants [6,7]. The practice parameter of the American Association of Child and Adolescent Psychiatry recommends electroconvulsive therapy (ECT) for adolescents in resistant cases of major depression [8]. However, ECT is less frequently used [4,9] due to cognitive side effects, prolonged seizure, and anesthetic complications [4,10]. As a result, there is an increasing interest in novel treatment approaches for major depression.

Repetitive transcranial magnetic stimulation (rTMS) is a relatively new treatment modality that is gaining attention in the treatment of various conditions afflicting adults [11-13]. Three large, multi-site, randomized, sham-controlled trials amongst medication-free depressed adults who failed to respond to antidepressant trials have demonstrated its efficacy in major depression [14-16]. A recent systematic review indicates a better response rate among adults with fewer previous failed antidepressant trials [17]. The response rate can be as high as $95.5 \%$ and a remission rate of $68.2 \%$ in the first episode [18].

The US FDA approved the use of rTMS for major depression and obsessive-compulsive disorder among adults who failed to respond to medications [13,19]. Adult literature indicates that the most common side-effects of rTMS are scalp tenderness and headache $[11,20]$. Serious side-effects such as seizure and treatmentemergent mania or hypomania are rare $[20,21]$. The adult literature generated a growing interest in investigating its role in depression among children and adolescents. The current systematic review explores the safety and efficacy of rTMS in the case of major depression among children and adolescents. 


\section{Review}

\section{Methods}

The systematic review aimed to collate various open-label studies, randomized controlled trials (RCTs), case reports, case series investigating the safety and efficacy of rTMS in treating major depression among children and adolescents (age $\leqslant 18$ ). Studies that included children and adults but focused on adults or did not present data of children and adolescents separately were not included. We searched the Medline and Cochrane databases and kept various MeSH keywords such as

"pediatric," "adolescent," "depression," "rTMS" in varying combinations. Additionally, we searched the reference lists of various articles and reviews using the same search engine and contacted other researchers to gather additional information about their published papers. We included literature in the English language published until November 2020. We excluded studies whose primary diagnosis was not major depression and those who used other variety of TMS. We have utilized the Joanna Briggs Institute quality appraisal tool to assess the quality of the included studies [22]. One of the authors (PM), in consultation with two other authors (SB and TP), selected the articles for the systematic review and followed the PRISMA guideline (Figure 1). We did not attempt a quantitative synthesis with the data due to the heterogeneity of studies and outcome parameters/assessment methods.
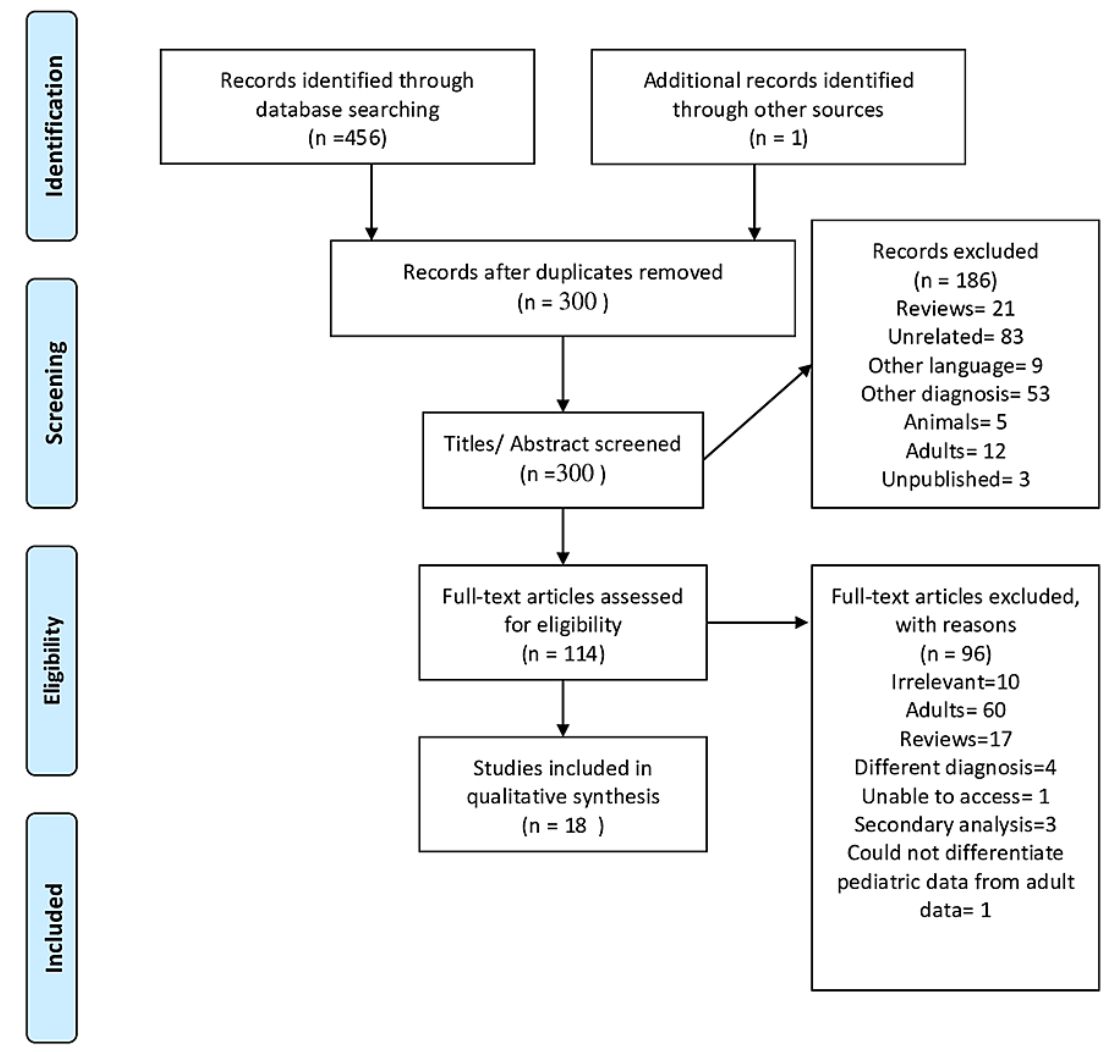

\section{FIGURE 1: PRISMA 2009 Flow Diagram}

PRISMA: Preferred Reporting Items for Systematic Reviews and Meta-Analyses. The image was obtained from Moher et al. [23].

\section{Results}

Using the various combinations of keywords, we retrieved 457 articles. Removal of duplicates resulted in 300 publications. All these 300 articles were screened based upon the titles and abstracts. We excluded 186 articles due to various reasons (as shown in Figure 1). We retrieved 114 full texts for eligibility amongst which, 96 were excluded based on relevance ( $\mathrm{N} 10)$, involving adults ( $\mathrm{N} 60)$, reviews (N 17), different diagnosis (N 4), unable to access (N 1), secondary analysis (N 3), and could not differentiate pediatric data from adult data (N 1). Finally, 18 articles, including 13 multi-subject trials and 5 case reports, were incorporated in this systematic review. We identified another three studies [24-26] that incorporated data from previous studies already included in this systematic review. We have discussed their findings.

Characteristics of the Studies 


\section{Cureus}

Tables 1 and 2 summarized the characteristics of the studies. The studies include open-label trials [27-32], case reports [33-37], case series [38-41], naturalistic studies [42], and randomized sham-controlled trial [43]. One study used emailed questionnaires to a Worldwide pool of rTMS centers [44]. The majority of the studies are from the USA [28-31,33-35,40,43]. The rest of the studies were from China [37,38,42], Israel [32], Finland [36], Canada [27,39], Australia [41], and across multiple nations [44]. The lowest age of the recipient of rTMS was ten $[40,42,43]$, but most of the patients were above 12 .

\begin{tabular}{|c|c|c|c|c|c|c|c|c|}
\hline Author & Study design & Rating scales & $\mathbf{N}$ (age) & $\begin{array}{l}\text { Number of } \\
\text { sessions }\end{array}$ & $\begin{array}{l}\text { Session duration (Sec per train, sec per } \\
\text { duration) }\end{array}$ & $\begin{array}{l}\text { Intensity } \\
\text { \%MT }\end{array}$ & $\begin{array}{l}\text { Reported side } \\
\text { effects }\end{array}$ & Efficacy/effectiveness \\
\hline $\begin{array}{l}\text { Croarkin et } \\
\text { al. [43] }\end{array}$ & $\begin{array}{l}\text { Randomized, } \\
\text { sham- } \\
\text { controlled }\end{array}$ & $\begin{array}{l}\text { HAM-D, MADRS, } \\
\text { CDRS-R, CGI-S, } \\
\text { QIDS-A-17-SR, } \\
\text { CSSR-S, YMRS }\end{array}$ & $\begin{array}{l}103(12-22) \\
\text { rTMS } \\
\text { group-48 } \\
\text { sham- } \\
\text { controlled } \\
\text { group-55 }\end{array}$ & 30 & $\begin{array}{l}75 \text { trains, } 10 \text { pulses per second over } 4 \\
\text { seconds, intertrain interval of } 26 \\
\text { seconds, } 10 \mathrm{~Hz}, 3000 \text { pulses per session }\end{array}$ & 120 & $\begin{array}{l}\text { Suicidal ideation, } \\
\text { classified as not } \\
\text { related to rTMS } \\
\text { headache, } \\
\text { nausea, facial } \\
\text { twitching }\end{array}$ & $\begin{array}{l}\text { Response rate is } 41.7 \% \text { (remission } \\
\text { rate } 29.2 \% \text { ) in the active treatment } \\
\text { group and } 36.4 \% \text { (remission rate } \\
29 \% \text { ) in sham-controlled group }\end{array}$ \\
\hline $\begin{array}{l}\text { Zhang et } \\
\text { al. [42] }\end{array}$ & $\begin{array}{l}\text { Naturalistic } \\
\text { study }\end{array}$ & HAM-D, HAM-A & $\begin{array}{l}42 \\
\text { adolescents } \\
\text { vs. } 75 \\
\text { adults (10- } \\
80)\end{array}$ & $11-20$ & $\begin{array}{l}80 \text { trains, } 30 \text { pulses per train, } 10 \mathrm{~Hz}, 12 \\
\text { seconds intertrain-interval, } 2400 \text { pulses } \\
\text { per session }\end{array}$ & 120 & $\begin{array}{l}\text { Transient } \\
\text { headache, } \\
\text { musculoskeletal } \\
\text { discomfort }\end{array}$ & $\begin{array}{l}\text { All age groups showed } \\
\text { improvement in both anxiety and } \\
\text { depression. Symptom } \\
\text { improvement more among } \\
\text { adolescents. Significant time-effect } \\
\text { was seen } 94.1 \% \text { of adolescents } \\
\text { responded, and } 88.2 \% \text { achieved } \\
\text { remission at the end of } 4 \text { weeks }\end{array}$ \\
\hline $\begin{array}{l}\text { MacMaster } \\
\text { et al. [27] }\end{array}$ & Open level & $\begin{array}{l}\text { K-SADS-PL, CDRS- } \\
\text { R, HAM-D, HAM-A, } \\
\text { BDI HAM-D, } \\
\text { Pediatric TMS safety } \\
\text { and tolerability } \\
\text { measure }\end{array}$ & $32(13-21)$ & 15 & $\begin{array}{l}40 \text { pulses over } 4 \text { seconds, } 10 \mathrm{~Hz} \text {; } \\
\text { intertrain interval, } 26 \text { s; } 75 \text { trains; } 3000 \\
\text { pulses }\end{array}$ & 120 & $\begin{array}{l}\text { Headache, mild } \\
\text { neck pain, } \\
\text { unpleasant } \\
\text { tingling, nausea, } \\
\text { lightheadedness }\end{array}$ & $\begin{array}{l}56 \% \text { responders responded and } \\
44 \% \text { achieved remission }\end{array}$ \\
\hline $\begin{array}{l}\text { Pan et al. } \\
\text { [38] }\end{array}$ & $\begin{array}{l}\text { Case series } \\
\text { from a } \\
\text { previous } \\
\text { double-blind } \\
\text { sham- } \\
\text { controlled } \\
\text { study }\end{array}$ & SCID BSI-CV MADRS & $\begin{array}{l}3(16,17, \\
15)\end{array}$ & 7 & $\begin{array}{l}120 \text { trains of } 5 \text { seconds dura tion at } 10 \\
\mathrm{~Hz} \text { with intertrain intervals of } 15 \text { seconds } \\
\text { (i.e., } 6000 \text { pulses per session }\end{array}$ & 100 & $\begin{array}{l}\text { Sleepiness, } \\
\text { hypomanic } \\
\text { symptoms }\end{array}$ & $\begin{array}{l}40-100 \% \text { improvement in BSI-CV } \\
\text { score, and } 14.63-47.83 \% \\
\text { improvement in MADRS score of } \\
\text { all three patients. Remission of } \\
\text { suicidal thought of one of the } \\
\text { patients }\end{array}$ \\
\hline $\begin{array}{l}\text { Wall et al. } \\
\text { [28] }\end{array}$ & $\begin{array}{l}\text { Open level } \\
\text { study }\end{array}$ & $\begin{array}{l}\text { KSADS-PL, CDRS-R, } \\
\text { QIDS-A17-SR, CSSR- } \\
\text { S, CGI-S, CGI-I }\end{array}$ & $\begin{array}{l}10(13.9- \\
17.4)\end{array}$ & 30 & $\begin{array}{l}\text { 4-second stimulus trains, } 10 \mathrm{HZ} \\
\text { separated by } 26 \text {-second intertrain } \\
\text { intervals, with } 3000 \text { magnetic pulses per } \\
\text { session }\end{array}$ & 120 & $\begin{array}{l}\text { Scalp pain, } \\
\text { worsening of } \\
\text { depression, } \\
\text { headaches, } \\
\text { dizziness, } \\
\text { musculoskeletal } \\
\text { discomfort, neck } \\
\text { stiffness, eye } \\
\text { twitching, and } \\
\text { nausea }\end{array}$ & $\begin{array}{l}60 \% \text { of participants responded. } \\
\text { The total mean score of CDRS-R, } \\
\text { QIDS-A17-SR, and CGI-S } \\
\text { significantly improved at } 20,30 \text {, } \\
\text { and at 6-month follow-up }\end{array}$ \\
\hline $\begin{array}{l}\text { Croarkin et } \\
\text { al. [29] }\end{array}$ & Open level & $\begin{array}{l}\text { K-SADS, SCID, } \\
\text { CDRS-R, ATHF }\end{array}$ & $10(13-17)$ & $\begin{array}{l}30 \text { Six } \\
\text { completed } \\
30, \text { and one } \\
\text { each } \\
\text { completed } \\
1,5,17 \text {, } \\
\text { and } 29 \\
\text { sessions }\end{array}$ & $\begin{array}{l}\text { 4-second stimulus trains, } 10 \mathrm{~Hz} \\
\text { separated by } 26-\text {-seconds intertrain } \\
\text { intervals, with } 3000 \text { magnetic pulses per } \\
\text { session }\end{array}$ & 120 & Unspecified & $\begin{array}{l}\text { 33.5\% improvement in CDRS-R } \\
\text { score post-treatment, and almost } \\
45.6 \% \text { improvement at } 6 \text { months } \\
\text { post-treatment }\end{array}$ \\
\hline $\begin{array}{l}\text { Yang et al. } \\
\text { [39] }\end{array}$ & Case series & $\begin{array}{l}\text { KSADS, HAMD, BDI, } \\
\text { HAMA }\end{array}$ & $6(15-21)$ & 15 & $\begin{array}{l}4 \text { seconds stimulus, intertrain interval } 26 \\
\text { seconds, } 10 \mathrm{~Hz}, 3000 \text { pulses per } \\
\text { treatment }\end{array}$ & $120 \%$ & $\begin{array}{l}\text { Mild scalp } \\
\text { discomfort, } \\
\text { headache }\end{array}$ & $\begin{array}{l}66 \% \text { responded } 68 \% \text { decrease in } \\
\text { HAMD score and } 84 \% \text { reduction in } \\
\text { BDI score } 78 \% \text { reduction of HAMA } \\
\text { score }\end{array}$ \\
\hline Wall et al. & Open level, & $\begin{array}{l}\text { KSADS-PL, CDRS-R, } \\
\text { ATHF, CAVLT-2, D- }\end{array}$ & $\begin{array}{l}18(13.9- \\
17.8) 14\end{array}$ & & 4 seconds stimulus duration, intertrain & & & Substantial improvement in \\
\hline
\end{tabular}




\section{Cureus}

\begin{tabular}{|c|c|c|c|c|c|c|c|c|}
\hline [30] & $\begin{array}{l}\text { prospective, } \\
\text { multi-center }\end{array}$ & $\begin{array}{l}\text { KEFS, trail-making } \\
\text { test }\end{array}$ & $\begin{array}{l}\text { completed } \\
\text { treatment }\end{array}$ & 30 & $\begin{array}{l}\text { interval of } 26 \text { seconds, } 10 \mathrm{~Hz}, 3000 \\
\text { stimuli per session }\end{array}$ & $120 \%$ & & depressive symptoms \\
\hline $\begin{array}{l}\text { Wall et al. } \\
\text { [31] }\end{array}$ & $\begin{array}{l}\text { Open level, } \\
\text { perspective }\end{array}$ & $\begin{array}{l}\text { K-SADS-PL, CDRS- } \\
\text { R, QIDS-A17, CGI-S } \\
\text { and CGI-I, CSSR-S, } \\
\text { Subjective Reactive } \\
\text { Questionnaire, } \\
\text { Adverse Event } \\
\text { Monitoring form }\end{array}$ & $\begin{array}{l}8(14.6- \\
17.8)\end{array}$ & 30 & $\begin{array}{l}4 \text { seconds stimulus duration, } 10 \mathrm{~Hz} \text {, } \\
\text { intertrain interval } 26 \text { seconds, } 75 \text { trains, } \\
3000 \text { stimulations per session }\end{array}$ & 120 & Scalp pain & $\begin{array}{l}\text { Almost } 50 \% \text { decrease in the mean } \\
\text { baseline CDRS and QIDS A17 from } \\
\text { baseline to at treatment } 30\end{array}$ \\
\hline $\begin{array}{l}\text { Croarkin et } \\
\text { al. [40] }\end{array}$ & Case series & $\begin{array}{l}\text { K-SADS-PL, CDRS- } \\
\text { R }\end{array}$ & $\begin{array}{l}2 \operatorname{cases}^{*}(10 \\
\text { and } 17)\end{array}$ & Unspecified & Unspecified & Up to 40 & Scalp pain & Unspecified \\
\hline $\begin{array}{l}\text { Bloch et al. } \\
\text { [32] }\end{array}$ & $\begin{array}{l}\text { Open level } \\
\text { study }\end{array}$ & $\begin{array}{l}\text { SCID, BDI, CDRS, } \\
\text { CGI-S, SIQ, SCARED, } \\
\text { CNTA }\end{array}$ & $9(16-18)$ & 14 & $\begin{array}{l}\text { 2-second trains, } 10 \mathrm{~Hz} \text {, intertrain interval } \\
58 \text { seconds, } 20 \text { stimuli, } 400 \text { stimuli per } \\
\text { session }\end{array}$ & $80 \%$ & Headache & $\begin{array}{l}\text { Subjects had lower depression, } \\
\text { anxiety, and no cognitive changes } \\
\text { or suicidal ideation }\end{array}$ \\
\hline $\begin{array}{l}\text { Loo et al. } \\
\text { [41] }\end{array}$ & $\begin{array}{l}\text { First two } \\
\text { cases from } \\
\text { double-blind } \\
\text { sham- } \\
\text { controlled } \\
\text { trial }\end{array}$ & $\begin{array}{l}\text { MDRAS, BDI, CGI-S, } \\
\text { CES- DC, RAVLT, } \\
\text { Weschler digit span } \\
\text { forward and } \\
\text { backward, TMT A } \\
\text { and B, COWAT }\end{array}$ & $2(16)$ & $\begin{array}{l}\text { Case } 1 \text { - } 29 \\
\text { Case } 2 \text { - } 27\end{array}$ & $\begin{array}{l}5 \text { seconds, } 10 \mathrm{~Hz}, 25 \text { seconds intertrain } \\
\text { interval, } 40 \text { trains, } 2000 \text { stimuli per } \\
\text { session }\end{array}$ & $110 \%$ & None & No cognitive changes \\
\hline $\begin{array}{l}\text { Walter et } \\
\text { al. [44] }\end{array}$ & $\begin{array}{l}\text { Emailed } \\
\text { questionnaire } \\
\text { to a } \\
\text { worldwide } \\
\text { pool of rTMS } \\
\text { centers }\end{array}$ & HAMD, BQ & $\begin{array}{l}3(16,17, \\
17)\end{array}$ & 10 & $\begin{array}{l}\text { One case with - } 2 \text { seconds stimulus } \\
\text { duration, } 10 \mathrm{~Hz} \text {, intertrain interval } 28 \\
\text { seconds, } 40 \text { trains, } 1600 \text { stimulations per } \\
\text { session. Two other cases - } 8 \text { seconds } \\
\text { stimulus duration, } 10 \mathrm{~Hz} \text {, intertrain } \\
\text { interval } 52 \text { seconds, } 20 \text { trains, } 1600 \\
\text { stimulus per session }\end{array}$ & & & \\
\hline
\end{tabular}

\section{TABLE 1: Study design, intervention, and efficacy multi-subject trials}

MINI: Mini International Neuropsychiatric Interview, MINI-KID: Mini International Neuropsychiatric Interview for Children, YMRS: Young Mania Rating Scale, K-SADS-PL: Kiddie Schedule for Affective Disorders and Schizophrenia Present and Lifetime version, CDRS-R: Children's Depression Rating Scale Revised, HAM-D: Hamilton Depression Rating Scale, HAM-A: Hamilton Anxiety Rating Scale, BDI: Beck Depression Inventory, C-SSRS: Columbia Suicide Severity Rating Scale, CDRS-R: Depression Rating Scale, Revised, BSI-CV: Beck Scale for Suicide Ideation-Chinese Version, MADRS: Montgomery-Asberg Depression Rating Scale, QIDS-A17-SR: Quick Inventory for Depressive Symptomatology Adolescent Seventeen Item Self Report, CGI-S: Clinical Global Impressions-Severity and CGI-I: Clinical Global Impressions-Improvement scales, ATHF: Antidepressant Treatment History Form, CAVLT-2: Children's Auditory Verbal Learning Test-2, D-KEFS: Delis-Kaplan Executive Function System, SIQ: Suicidal Ideation Questionnaire, SCARED: Screen for Child Anxiety-Related Disorders questionnaire, CNTA: Cambridge Neuropsychological Test Automated Battery, CES-DC: Centre for Epidemiological Studies-Depression Scale for Child, RAVLT: Rey Auditory Verbal Learning Test, TMT: Trail Making Test A and B, COWAT: Controlled Oral Word Association Test - letter and category, BQ: Beck's Questionnaire.

*The authors presented three cases in the original article. However, one out of the three cases was a healthy control. The healthy control was excluded from this systematic review.

Pediatric TMS Safety and Tolerability Measure. 


\section{Cureus}

\begin{tabular}{|c|c|c|c|c|c|c|c|}
\hline Author & $\begin{array}{l}\text { Rating } \\
\text { scales }\end{array}$ & $\begin{array}{l}\text { Age } \\
\text { (years) }\end{array}$ & $\begin{array}{l}\text { Number } \\
\text { of } \\
\text { sessions }\end{array}$ & $\begin{array}{l}\text { Session duration (second per train, } \\
\text { second per duration) }\end{array}$ & $\begin{array}{l}\text { Intensity } \\
\% \mathrm{MT}\end{array}$ & $\begin{array}{l}\text { Reported side } \\
\text { effects }\end{array}$ & Efficacy/effectiveness \\
\hline $\begin{array}{l}\text { Cullen et al. } \\
\text { [33] }\end{array}$ & None & 17 & 8 & $\begin{array}{l}\text { 2-second trains, } 18 \mathrm{~Hz}, 55 \text {, total } 1980 \\
\text { pulses }\end{array}$ & $85-120$ & $\begin{array}{l}\text { Scalp pain } \\
\text { Generalized, } \\
\text { tonic-clonic } \\
\text { seizure }\end{array}$ & Unspecified \\
\hline $\begin{array}{l}\text { Cristancho } \\
\text { et al. [34] }\end{array}$ & $\begin{array}{l}\text { No } \\
\text { rating } \\
\text { scale } \\
\text { used }\end{array}$ & 15 & $\begin{array}{l}10 \text { at } \\
\text { RDLPFC } \\
26 \text { at } \\
\text { LDLPFC }\end{array}$ & $\begin{array}{l}1 \mathrm{~Hz}, 10 \text { seconds on, and } 10-15 \text { seconds } \\
\text { off. The pulse increased from } 150 \text { PPS and } \\
\text { increased to } 300 \text { PPS by the second week } \\
\text { over RDLPFC and then } 300-600 \text { PPS by } \\
\text { the fourth week over LDLPFC }\end{array}$ & 90 & $\begin{array}{l}\text { Mild } \\
\text { headaches, } \\
\text { jaw twitch, and } \\
\text { transient } \\
\text { dizziness }\end{array}$ & $\begin{array}{l}\text { Improvement in mood, } \\
\text { less tearfulness }\end{array}$ \\
\hline $\begin{array}{l}\text { Segev et al. } \\
\text { [35] }\end{array}$ & $\begin{array}{l}\text { BDI-II, } \\
\text { CDRS- } \\
\text { R }\end{array}$ & 17 & 20 & $\begin{array}{l}\text { 4-second stimulus, intertrain interval } 30 \\
\text { seconds, } 10 \mathrm{~Hz}, 1680 \text { pulses per treatment }\end{array}$ & 100 & $\begin{array}{l}\text { Headache, } \\
\text { scalp pain, and } \\
\text { scalp burning }\end{array}$ & $\begin{array}{l}\text { Improvement in anxiety } \\
\text { from } 46 \text { to } 25 \text { score in } \\
\text { SCARED. No } \\
\text { improvement in BDI-II } \\
\text { and CDRS-R scores. } \\
\text { SIQ score improved } \\
\text { transiently. }\end{array}$ \\
\hline $\begin{array}{l}\text { Chiramberro } \\
\text { et al. [36] }\end{array}$ & SCID & 16 & 12 & $\begin{array}{l}\text { 5-second stimulus duration, } 10 \mathrm{~Hz}, 60 \\
\text { trains, intertrain interval - } 25 \text { seconds, } 3000 \\
\text { stimuli per session }\end{array}$ & Unspecified & Seizure & Unspecified \\
\hline $\begin{array}{l}\text { Hu et al. } \\
\text { [37] }\end{array}$ & & 15 & 1 & $\begin{array}{l}\text { 4-second stimulus duration, } 10 \mathrm{~Hz} \text {, } \\
\text { intertrain interval } 26 \text { seconds, } 20 \text { trains per } \\
\text { session, the cumulative number of daily } \\
\text { pulses } 800\end{array}$ & 80 & $\begin{array}{l}\text { The } \\
\text { generalized } \\
\text { tonic-clonic } \\
\text { seizure } \\
\text { followed by } \\
\text { hypomanic } \\
\text { symptoms } \\
\text { lasting for 8-9 } \\
\text { hours }\end{array}$ & Unspecified \\
\hline
\end{tabular}

TABLE 2: Study design, intervention, and efficacy case reports

RDLPFC: right dorsolateral prefrontal cortex, LDLPFC: left dorsolateral prefrontal cortex, PPS: pulses per session, SCID: severe combined immunodeficiency.

The sample size ranged from 1 (case reports) to 103 [43]. The case series by Croarkin et al. presented three cases [40]. However, one of the three cases was a healthy control. So, we have included two out of these three cases in this systematic review. Similarly, the case series by Walter et al. presented seven cases. However, we included three of them as the other four subjects had other primary diagnoses such as bipolar disorder and schizophrenia [44]. All the multi-subject trials allowed comorbid anxiety disorder, dysthymia, attention deficit hyperactivity disorder (ADHD) but excluded schizophrenia, bipolar disorder, substance use disorder, post-traumatic stress disorder (PTSD), intellectual disability, pervasive developmental disorders, and eating disorders. One study included comorbid substance abuse [32], and another one allowed the inclusion of bipolar type II [42]. Zhang et al. included "mood or anxiety disorder" and thus, incorporated other diagnoses such as bipolar type II, dysthymia, generalized anxiety disorder [42]. No change in psychotherapy was allowed a few weeks before and during the trial [30-32]. Some studies have excluded patients with suicide attempts in the previous six months [28,31] or with "suicidal propensity" [42].

Most of the studies included treatment-resistant depression $[25-32,39,43]$ and established the diagnosis by Kiddie Schedule for Affective Disorder and Schizophrenia [27-31,39,40]. Others utilized Structured Clinical Interviews based on the Diagnostic and Statistical Manual of Mental Disorders, fourth edition (DSM-IV) [32,36,38]; clinical interviews [35,42,43], Mini International Neuropsychiatric Interview for children, or Mini Neuropsychiatric Interview [43]. One of the studies used the World Health Organization International Classification of Disease - version 10 [37].

The outcomes were measured using Hamilton Depression Rating Scale (HAM-D) and Hamilton Anxiety Rating Scale (HAM-A) [27,39,42], HAM-D [43], Montgomery-Asberg Depression Rating Scale [38,41,43], Children’s Depression Rating Scale, Revised [28,29,31,32,35,43], Quick Inventory for Depressive 


\section{Cureus}

Symptomatology Adolescent Seventeen Item Self Report [28,31,43], Clinical Global Impressions-Severity $[28,31,32,41,43]$, Screen for Child Anxiety-Related Disorders questionnaire [32,35], Beck Depression Inventory [32,35,39,41], Beck Scale for Suicide Ideation-Chinese Version [38], Columbia Suicide Severity Rating Scale [28,31,43], Suicidal Ideation Questionnaire [32,35], Mini International Neuropsychiatric Interview [43], Mini International Neuropsychiatric Interview for Children [43], and Young Mania Rating Scale [43].

Antidepressant Use Among the Included Studies

The antidepressant use of the included studies is summarized in Tables 3 and 4 . All the studies, except Croarkin et al., allowed antidepressant medications [43]. The studies have included patients with a history of one [27-30,39], two [32] to several failed antidepressant trials [28,33,40,43]. Pan et al. included either drug naïve or patients not taking antidepressants for nearly two weeks [38]. The majority of the studies have specified no change in antidepressant dosages during the trial [28-32,42]. Participants of some studies did not take any antipsychotics, mood stabilizers, benzodiazepines, stimulants, tricyclic antidepressants, or bupropion during TMS treatment [28,31]. 


\section{Cureus}

\begin{tabular}{|c|c|c|c|c|}
\hline Author & Antidepressant use & Previous treatment failure & $\begin{array}{l}\text { Psychosocial } \\
\text { intervention used }\end{array}$ & $\begin{array}{l}\text { Change of } \\
\text { dosages } \\
\text { allowed }\end{array}$ \\
\hline $\begin{array}{l}\text { Croarkin et } \\
\text { al. [43] }\end{array}$ & None & Yes & $\begin{array}{l}\text { No change in } \\
\text { psychosocial } \\
\text { interventions }\end{array}$ & N/A \\
\hline $\begin{array}{l}\text { Zhang et } \\
\text { al. [42] }\end{array}$ & $\begin{array}{l}\text { All used one type of } \\
\text { antidepressant treatment } \\
\text { during rTMS along with other } \\
\text { medications }\end{array}$ & $\begin{array}{l}\text { Unspecified but excluded patients who } \\
\text { were taking more than one antidepressant }\end{array}$ & Unspecified & No \\
\hline $\begin{array}{l}\text { MacMaster } \\
\text { et al. [27] }\end{array}$ & $\begin{array}{l}\text { Psychotropic medications } \\
\text { use were allowed if stable for } \\
6 \text { weeks } 31.25 \% \text { were not on } \\
\text { psychotropics }\end{array}$ & At least two previous failed medication trials & Unspecified & $\begin{array}{l}\text { No unless } \\
\text { medically } \\
\text { necessary }\end{array}$ \\
\hline $\begin{array}{l}\text { Pan et al. } \\
{[38]}\end{array}$ & $\begin{array}{l}\text { Either drug naïve or was not } \\
\text { taking antidepressants for } 2 \\
\text { weeks prior to the study }\end{array}$ & $\begin{array}{l}\text { All three patients took an antidepressant in } \\
\text { the past for an unspecified dose and } \\
\text { duration }\end{array}$ & Unspecified & N/A \\
\hline $\begin{array}{l}\text { Wall et al. } \\
{[28]}\end{array}$ & All used either SSRI or SNRI & $\begin{array}{l}\text { At least one prior medication trial failure as } \\
\text { defined by ATHF. Study participants had a } \\
\text { mean of } 4.0 \text { prior failed medication trial (SD } \\
\text { 2.1). }\end{array}$ & $\begin{array}{l}\text { No change in } \\
\text { psychotherapy treatment } \\
\text { was allowed within the } \\
\text { last } 4 \text { weeks of treatment }\end{array}$ & No \\
\hline $\begin{array}{l}\text { Croarkin et } \\
\text { al. [29] }\end{array}$ & All were on antidepressants & At least one prior failed medication trials & Unspecified & No \\
\hline $\begin{array}{l}\text { Yang et al. } \\
\text { [39] }\end{array}$ & Unspecified & At least one failed trial & Unspecified & Unspecified \\
\hline $\begin{array}{l}\text { Wall et al. } \\
{[30]}\end{array}$ & All were on antidepressants & At least one prior failed trial & $\begin{array}{l}\text { No change in } \\
\text { psychotherapy or } \\
\text { therapist }\end{array}$ & No \\
\hline $\begin{array}{l}\text { Wall et al. } \\
\text { [31] }\end{array}$ & Yes & Yes - at least two failed trials & $\begin{array}{l}\text { No change in } \\
\text { psychotherapy or } \\
\text { therapist in } 4 \text { weeks prior } \\
\text { to rTMS }\end{array}$ & No \\
\hline $\begin{array}{l}\text { Croarkin et } \\
\text { al. [40] }\end{array}$ & Case 1 - none; Case 2 - yes & Case 1 - none; case 2 - multiple & Unspecified & No \\
\hline $\begin{array}{l}\text { Bloch et al. } \\
\text { [32] }\end{array}$ & Yes & $\begin{array}{l}\text { Failure of one course of psychotherapy and } \\
\text { two courses of medication trials } 8 \text { weeks } \\
\text { each, with at least one of the } \\
\text { antidepressants being Fluoxetine }\end{array}$ & $\begin{array}{l}\text { No change in therapy } \\
\text { within } 5 \text { weeks before } \\
\text { starting of rTMS }\end{array}$ & No \\
\hline $\begin{array}{l}\text { Loo et al. } \\
\text { [41] }\end{array}$ & $\begin{array}{l}\text { Case } 1 \text { - unspecified; case } 2 \text { - } \\
\text { yes }\end{array}$ & $\begin{array}{l}\text { Case } 1 \text { - failure of one course of } \\
\text { psychotherapy; case } 2 \text { - one course of } \\
\text { psychotherapy, two medication trials }\end{array}$ & Unspecified & $\begin{array}{l}\text { Case } 1 \text { - } \\
\text { unspecified; } \\
\text { Case } 2 \text { - no }\end{array}$ \\
\hline $\begin{array}{l}\text { Walter et } \\
\text { al. [44] }\end{array}$ & No & Unspecified & Unspecified & Unspecified \\
\hline
\end{tabular}

\section{TABLE 3: Antidepressant use and psychosocial intervention during the trial multi-subject trials}

SSRI: selective serotonin reuptake inhibitor; SNRI: serotonin and norepinephrine reuptake inhibitor, ATHF: Antidepressant Treatment History Form, TCA: tricyclic antidepressants. 


\section{Cureus}

\begin{tabular}{|c|c|c|c|c|}
\hline Author & $\begin{array}{l}\text { Antidepressant } \\
\text { use }\end{array}$ & $\begin{array}{l}\text { Previous treatment } \\
\text { failure }\end{array}$ & $\begin{array}{l}\text { Psychosocial intervention } \\
\text { used }\end{array}$ & $\begin{array}{l}\text { Change of dosages } \\
\text { allowed }\end{array}$ \\
\hline Cullen et al. [33] & None & Multiple failed trials & Unspecified & N/A \\
\hline Cristancho et al. [34] & Yes & Multiple failed trials & Unspecified & N/A \\
\hline Segev et al. [35] & Yes & Yes & Unspecitıed & Unspecitied \\
\hline $\begin{array}{l}\text { Chiramberro et al. } \\
\text { [36] }\end{array}$ & Yes & Yes & Unspecified & Unspecified \\
\hline Hu et al. [37] & Yes on sertraline & Unspecified & Unspecified & Unspecified \\
\hline
\end{tabular}

TABLE 4: Antidepressant use and psychosocial intervention during the trial case reports

Administration of rTMS

All studies delivered rTMS over the left dorsolateral prefrontal cortex (LDLPFC), except one study by Cristancho et al. that delivered it in both right and left DLPFC [34]. Most of them utilized the " $5 \mathrm{~cm}$ rule" for localization of LDLPFC. Most of the studies used a figure of eight coil [27,34-39,41,42], and others used circular coil [32] or H1 coil [33]. The number of sessions varied from 1 [37,40] to 30 [28-31,43]. Most of the studies delivered rTMS at a frequency of $10 \mathrm{~Hz}$, except two studies, Cullen et al. (18 Hz) and Cristancho et al. $(1 \mathrm{~Hz})[33,34]$. Seven studies delivered 3000 stimuli per session $[27-31,36,39,43]$. The majority of the studies used $120 \%$ of motor threshold (MT) [27-31,39,42,43] and others varied from $40 \%$ to $120 \%$, such as $100 \%$ [35,38], 90\% [34], 80\% [37], 80\% [32], 110\% [41], and 90-110\% [44].

\section{Efficacy/Effectiveness}

The majority of the studies found that rTMS is a safe and effective add-on treatment for child and adolescent depression [27-32,34,38,39,42]. The improvement started as early as one [32,38] to two weeks $[28,42]$. Treatment benefits were noted at six months [28-31] to one year [32] post-trials. Croarkin et al. reported an improvement of 33.5\% in CDRS-R scores and almost $45.6 \%$ improvement at six months post-treatment [29]. A similar rate of improvement (33\%) was reported by Bloch et al. [32]. Wall et al. noted improvement among $60 \%$ of the study subjects [28]. Zhang et al. compared 42 adolescents with 75 adults and found a response rate of $94.1 \%$ and a remission rate of $88.2 \%$ among adolescents [42]. The older group had a less impressive improvement, higher baseline depression and anxiety scores, and shorter follow-up. A secondary analysis by Zhang et al. indicated that add-on rTMS improved depression and somatic and psychic anxiety among adolescents [24]. Sonmez et al. pooled data from three previous studies [28,30,31] and found that rTMS may improve hypersomnia in addition to its antidepressant action [26].

\section{Side Effects}

The majority of the studies reported mild side-effects such as scalp tenderness and headache [27,28,3135,39,42-44], musculoskeletal discomfort [28,42], neck-pain and stiffness [27,28]. The other side effects that were reported include eye-twitching [28], eye pain [43], facial twitch [43], jaw-twitching [34], nausea [27,28,43], tingling sensation [27], and lightheadedness/dizziness [27,28,34]. There are three case reports of treatment-emergent seizures [33,36,37] and one case series of intolerable headaches [40] leading to the termination of the procedure. There is one case report of emergent hypomanic symptoms [38]. No cognitive decline was reported $[30,31]$.

\section{Suicidal Ideation}

Several studies have assessed suicidal behavior and found no worsening of symptoms during rTMS. Croarkin et al. reported that 4 out of 103 patients developed suicidal ideation, but none were related to rTMS treatment [43]. Wall et al. reported 8 out of 10 subjects had suicidal ideation before the study, two subjects experienced worsening suicidal thoughts during the trial [28]. One subject had self-injurious behavior at six months follow up. In another study, subjects with suicidal thoughts at baseline reported improvement with treatment [31]. At the end of six months, one patient had self-mutilating behavior, and another patient needed hospitalization, which was unrelated to rTMS. In a pooled data analysis of the above studies [28,31], the suicidal ideation at baseline and at the end of the study period was $63.16 \%$ and $16.67 \%$, respectively [25]. In another case series by Pan et al., one out of three subjects experienced remission of suicidal ideation during rTMS [38]. Segev et al. reported a case where suicidality was transiently improved, followed by deterioration, and finally plateaued [35]. Bloch et al. also found out that suicidal ideation was not affected by the stage of rTMS treatment [32]. 


\section{Discussion}

To the best of our knowledge, our systematic review is the only one that has incorporated the data of more than 250 patients. Our findings support the results of the previous systematic reviews on the topic $[45,46]$. Previous systematic reviews on this topic have been criticized for lacking a controlled group in the included studies and the relatively smaller number of total subjects in the included studies $[45,46]$. In our systematic review, the addition of the only available RCT with a sham-control group improved the quality of the evidence of efficacy by utilizing a comparison group that approximates a placebo group [47]. Our systematic review included the three recent studies with larger sample sizes than previous articles $[27,42,43]$. Although better quality studies are still needed, there is a strong indication that rTMS is a safe and useful add-on, but not a stand-alone treatment for child and adolescent depression that has not responded to at least one antidepressant treatment. Twelve out of 18 included studies found out that rTMS could be an effective treatment for major depression among children and adolescents.

Studies showing reduced depressive symptoms have most commonly delivered rTMS in $10 \mathrm{~Hz}$ frequency, $120 \%$ MT, four seconds train, and 3000 stimuli per session for 30 sessions. One of the significant advantages of rTMS is that the patient can be monitored multiple times a week. Moreover, the side- effects are mild and self-limiting. Suicidality and treatment-emergent manic or hypomanic symptoms appear to be rare with rTMS in this age group. The side effects reported in the majority of the studies are mild and selflimiting. Out of the three case reports demonstrating the development of seizure spells in the context of rTMS, one delivered rTMS in a somewhat different protocol from the other studies, such as they used twosecond trains (instead of the usual four-second trains used by other authors), used $18 \mathrm{~Hz}$ frequency instead of $10 \mathrm{~Hz}$ frequency, and delivered a total of 1980 pulses per session [33]. The other case report of seizure spell involved the use of $75 \mathrm{mg}$ olanzapine (which was later corrected by the author as $7.5 \mathrm{mg}$ ) and a blood alcohol concentration of $0.20 \%$ at the time of the seizure episode [36]. The only one case report of the emergence of hypomanic symptoms has used 6000 pulses per session [38]. Such a low occurrence of significant side effects has also been reported in other non-invasive brain stimulation studies involving children and adolescents [48-50].

According to the University of Oxford Centre for Evidence-Based Medicine published criteria to determine the evidence levels, the sham-controlled RCT demonstrated level 1 evidence that rTMS is safe but is not better than placebo as a stand-alone treatment for resistant depression among children and adolescents [23]. Nevertheless, $41.7 \%$ responded, and $29.2 \%$ achieved remission with rTMS [43]. Seven open-label studies showed level 2 evidence for efficacy [27-32,42]. Multiple case series and case reports showed a level 4 degree of evidence of efficacy [34-41,44]. The study's treatment response rate by Zhang et al. seems to be an outlier. It could be because of the naturalistic study design or having a somewhat different inclusion and exclusion criteria [42]. Further, the study's comparison groups were different from each other in significant parameters such as the course of illness, baseline HAM-D score [42]. The studies that did not find rTMS as a useful tool for the treatment of depression have either used it as a stand-alone treatment [43] or comprise of only case reports demonstrating side-effects that led to premature termination of treatment [36,37,40].

We have used Joanna Briggs Institute Critical Appraisal Tools to assess the quality of the included studies (see Appendix) [22]. The overall quality of the studies is average except for Croarkin et al. which seems to have high [43]. rTMS can still be considered a relatively novel treatment option for minors and could have contributed to the included studies' relatively small sample sizes. Moreover, almost all the studies have used well-validated diagnostic tools and rating scales to provide objective data of the treatment response. All the studies attempted to use rTMS only in the context of previous treatment failures. Still, the existing database's main drawbacks are the studies' limited sample size and mostly open-label study designs. The overall quality of the existing studies is low. The other limitations are heterogeneity in the inclusion criteria, the number of previously failed antidepressant trials, various parameters, and the number of rTMS sessions. The heterogeneity mentioned above could explain some varied treatment responses in the reported studies. There is a strong need to conduct RCTs involving large study subjects to explore the efficacy of rTMS as an add-on treatment in child and adolescent major depression. There is a need to explore the stage of treatment of Major Depression when the addition of rTMS to antidepressant treatment could lead to the best possible treatment outcome in terms of its utility as an add-on agent for treatment. This is especially true given the favorable side effects reported in the previously published studies. Although it is challenging to draw a sweeping conclusion at this time, rTMS has a strong potential in treating depression among children and adolescents, particularly among adolescents.

\section{Conclusions}

Major depression among children and adolescents is associated with a high non-response rate with traditional treatment. rTMS can be a useful add-on treatment for child and adolescent major depression, not responding to at least one antidepressant. The existing database is limited by the limited number of RCTs. Despite limitations, most of the studies indicate that rTMS is generally safe among children and adolescents and carries a minimal risk of seizure and treatment-emergent hypomanic symptoms. rTMS is not more effective than placebo as a stand-alone treatment of resistant major depression among children and adolescents. 


\section{Cureus}

\section{Appendices}

\begin{tabular}{|c|c|c|c|c|c|c|c|c|}
\hline & Q1 & Q2 & Q3 & Q4 & Q5 & Q6 & Q7 & Q8 \\
\hline Cullen et al. [33] & - & - & - & + & - & - & + & + \\
\hline Cristancho et al. [34] & + & + & + & - & + & - & + & + \\
\hline Segev et al. [35] & + & + & + & + & + & + & + & + \\
\hline Chiramberro et al. [36] & - & + & + & + & + & - & + & - \\
\hline Hu et al. [37] & - & - & - & - & + & + & + & + \\
\hline
\end{tabular}

\section{TABLE 5: Joanna Briggs institute quality appraisal tool for case reports}

Yes $=+$, No $=-$, Unclear $=$ ?, Not applicable $=$ NA.

Q1. Were the patient's demographic characteristics clearly described? Q2. Was the patient's history clearly described and presented as a timeline? Q3. Was the current clinical condition of the patient on presentation clearly described? Q4. Were diagnostic tests or assessment methods and the results clearly described? Q5. Was the intervention(s) or treatment procedure(s) clearly described? Q6. Was the post-intervention clinical condition clearly described? Q7. Were adverse events (harms) or unanticipated events identified and described? Q8. Does the case report provide takeaway lessons?

\begin{tabular}{|c|c|c|c|c|c|c|c|c|c|}
\hline & Q1 & Q2 & Q3 & Q4 & Q5 & Q6 & Q7 & Q8 & Q9 \\
\hline Zhang et al. [42] & + & - & + & - & + & + & + & + & + \\
\hline MacMaster et al. [27] & + & + & + & - & + & + & + & + & + \\
\hline Wall et al. [28] & + & + & + & - & + & + & + & + & + \\
\hline Croarkin et al. [29] & + & + & + & - & + & + & + & + & + \\
\hline Wall et al. [30] & + & + & + & - & + & + & + & + & + \\
\hline Wall et al. [31] & + & + & + & - & + & + & + & + & + \\
\hline Bloch, 2008 [32] & + & + & + & - & + & + & + & + & + \\
\hline
\end{tabular}

\section{TABLE 6: Joanna Briggs institute quality appraisal tool for quasi-experimental studies (non-} randomized experimental studies)

Yes $=+$, No= -, Unclear $=$ ?, Not applicable $=$ NA.

Q1. Is it clear in the study what is the 'cause' and what is the effect' (i.e., there is no confusion about which variable comes first)? Q2. Were the participants included in any comparisons similar? Q3. Were the participants included in any comparisons receiving similar treatment/care, other than the exposure or intervention of interest? Q4. Was there a control group? Q5. Were there multiple measurements of the outcome both pre and post the intervention/exposure? Q6. Was follow-up complete and if not, were differences between groups in terms of their follow-up adequately described and analyzed? Q7. Were the outcomes of participants included in any comparisons measured in the same way? Q8. Were outcomes measured in a reliable way? Q9. Was appropriate statistical analysis used? 


\section{Cureus}

\begin{tabular}{|c|c|c|c|c|c|c|c|c|c|c|c|c|c|}
\hline & Q1 & Q2 & Q3 & Q4 & Q5 & Q6 & Q7 & Q8 & Q9 & Q10 & Q11 & Q12 & Q13 \\
\hline Croarkin et al. [43] & + & + & + & + & + & + & + & + & $?$ & + & + & + & + \\
\hline
\end{tabular}

TABLE 7: Joanna Briggs institute quality appraisal tool for randomized controlled trials

Yes $=+$, No $=-$, Unclear $=$ ?, Not applicable $=$ NA.

Q1. Was true randomization used for the assignment of participants to treatment groups? Q2. Was allocation to treatment groups concealed? Q3. Were treatment groups similar at the baseline? Q4. Were participants blind to treatment assignment? Q5. Were those delivering treatment blind to treatment assignment? Q6. Were outcomes assessors blind to treatment assignment? Q7. Were treatment groups treated identically other than the intervention of interest? Q8. Was follow-up complete and if not, were differences between groups in terms of their follow-up adequately described and analyzed? Q9. Were participants analyzed in the groups to which they were randomized? Q10. Were outcomes measured in the same way for treatment groups? Q11. Were outcomes measured in a reliable way? Q12. Was appropriate statistical analysis used? Q13. Was the trial design appropriate, and any deviations from the standard RCT design (individual randomization, parallel groups) accounted for in the conduct and analysis of the trial?

\begin{tabular}{|c|c|c|c|c|c|c|c|c|c|c|}
\hline & Q1 & Q2 & Q3 & Q4 & Q5 & Q6 & Q7 & Q8 & Q9 & Q10 \\
\hline Pan et al. [38] & + & + & + & - & - & - & + & + & - & NA \\
\hline Yang et al. [39] & + & + & + & ? & + & - & - & + & - & + \\
\hline Croarkin et al. [40] & - & + & + & - & - & - & + & + & - & NA \\
\hline Loo et al. [41] & + & + & + & + & - & - & + & + & - & ? \\
\hline Walter et al. [44] & - & + & + & - & - & - & - & + & - & - \\
\hline
\end{tabular}

\section{TABLE 8: Joanna Briggs institute quality appraisal tool for case series}

Yes $=+$, No $=-$, Unclear $=$ ?, Not applicable $=$ NA.

Q1. Were there clear criteria for inclusion in the case series? Q2. Was the condition measured in a standard, reliable way for all participants included in the case series? Q3. Were valid methods used for the identification of the condition for all participants included in the case series? Q4. Did the case series have consecutive inclusion of participants? Q5. Did the case series have complete inclusion of participants? Q6. Was there clear reporting of the demographics of the participants in the study? Q7. Was there clear reporting of clinical information of the participants? Q8. Were the outcomes of follow-up results of cases clearly reported? Q9. Was there clear reporting of the presenting site(s)/clinic(s) demographic information? Q10. Was statistical analysis appropriate?

\section{Additional Information \\ Disclosures}

Conflicts of interest: In compliance with the ICMJE uniform disclosure form, all authors declare the following: Payment/services info: All authors have declared that no financial support was received from any organization for the submitted work. Financial relationships: All authors have declared that they have no financial relationships at present or within the previous three years with any organizations that might have an interest in the submitted work. Other relationships: Dr. Pradipta Majumder is an rTMS provider. However, this has not have influenced the outcome ot the systematic review. Dr. Perera is an rTMS provider. However, this has not have influenced the outcome of the systematic review. No conflict of interest relevant for other authors.

\section{Acknowledgements}

We are indebted to Dr. Paul Croarkin, D.O., M.S. for his timely support in providing additional materials and full-texts of some published papers.

\section{References}

1. Mojtabai R, Olfson M, Han B: National trends in the prevalence and treatment of depression in adolescents and young adults. Pediatrics. 2016, 138:e20161878. 10.1542/peds.2016-1878

2. Clayborne ZM, Varin M, Colman I: Systematic review and meta-analysis: adolescent depression and longterm psychosocial outcomes. J Am Acad Child Adolesc Psychiatry. 2019, 58:72-9. 10.1016/j.jaac.2018.07.896

3. March J, Silva S, Petrycki S, et al.: Fluoxetine, cognitive-behavioral therapy, and their combination for adolescents with depression: Treatment for Adolescents With Depression Study (TADS) randomized 
controlled trial. JAMA. 2004, 292:807-20. 10.1001/jama.292.7.807

4. Lima NN, Nascimento VB, Peixoto JA, et al.: Electroconvulsive therapy use in adolescents: a systematic review. Ann Gen Psychiatry. 2013, 12:17. 10.1186/1744-859X-12-17

5. Zhou X, Michael KD, Liu Y, et al.: Systematic review of management for treatment-resistant depression in adolescents. BMC Psychiatry. 2014, 14:340. 10.1186/s12888-014-0340-6

6. Clark MS, Jansen KL, Cloy JA: Treatment of childhood and adolescent depression. Am Fam Physician. 2012, $86: 442-8$.

7. Chung SW, Hoy KE, Fitzgerald PB: Theta-burst stimulation: a new form of TMS treatment for depression? . Depress Anxiety. 2015, 32:182-92. 10.1002/da.22335

8. Ghaziuddin N, Kutcher SP, Knapp P, et al.: Practice parameter for use of electroconvulsive therapy with adolescents. J Am Acad Child Adolesc Psychiatry. 2004, 43:1521-39. 10.1097/01.chi.0000142280.87429.68

9. Benson NM, Seiner SJ: Electroconvulsive therapy in children and adolescents: clinical indications and special considerations. Harv Rev Psychiatry. 2019, 27:354-8. 10.1097/HRP.0000000000000236

10. Wang S, Yang C, Jia J, Zhou Y, Zheng Y: Use of electroconvulsive therapy in adolescents with schizophrenia in China. Child Adolesc Psychiatry Ment Health. 2018, 12:49. 10.1186/s13034-018-0254-Z

11. McClintock SM, Reti IM, Carpenter LL, et al.: Consensus recommendations for the clinical application of repetitive transcranial magnetic stimulation (rTMS) in the treatment of depression. J Clin Psychiatry. 2018, 79:16cs10905. 10.4088/JCP.16cs10905

12. Trevizol AP, Shiozawa P, Cook IA, et al.: Transcranial magnetic stimulation for obsessive-compulsive disorder: an updated systematic review and meta-analysis. J ECT. 2016, 32:262-6. 10.1097/YCT.0000000000000335

13. Cole JC, Green Bernacki C, Helmer A, Pinninti N, O'reardon JP: Efficacy of transcranial magnetic stimulation (TMS) in the treatment of schizophrenia: a review of the literature to date. Innov Clin Neurosci. 2015, 12:12-9.

14. Levkovitz Y, Isserles M, Padberg F, et al.: Efficacy and safety of deep transcranial magnetic stimulation for major depression: a prospective multicenter randomized controlled trial. World Psychiatry. 2015, 14:64-73. 10.1002/wps.20199

15. George MS, Lisanby SH, Avery D, et al.: Daily left prefrontal transcranial magnetic stimulation therapy for major depressive disorder: a sham-controlled randomized trial. Arch Gen Psychiatry. 2010, 67:507-16. 10.1001/archgenpsychiatry.2010.46

16. O'Reardon JP, Peshek AD, Romero R, Cristancho P: Neuromodulation and transcranial magnetic stimulation (TMS): a 21st century paradigm for therapeutics in psychiatry. Psychiatry (Edgmont). 2006, 3:30-4.

17. Voigt J, Carpenter L, Leuchter A: A systematic literature review of the clinical efficacy of repetitive transcranial magnetic stimulation (rTMS) in non-treatment resistant patients with major depressive disorder. BMC Psychiatry. 2019, 19:13. 10.1186/s12888-018-1989-z

18. Wang YM, Li N, Yang LL, et al.: Randomized controlled trial of repetitive transcranial magnetic stimulation combined with paroxetine for the treatment of patients with first-episode major depressive disorder. Psychiatry Res. 2017, 254:18-23. 10.1016/j.psychres.2017.04.005

19. Perera T, George MS, Grammer G, Janicak PG, Pascual-Leone A, Wirecki TS: The clinical TMS society consensus review and treatment recommendations for TMS therapy for major depressive disorder. Brain Stimul. 2016, 9:336-4. 10.1016/j.brs.2016.03.010

20. Taylor R, Galvez V, Loo C: Transcranial magnetic stimulation (TMS) safety: a practical guide for psychiatrists. Australas Psychiatry. 2018, 26:189-92. 10.1177/1039856217748249

21. Dolberg OT, Schreiber S, Grunhaus L: Transcranial magnetic stimulation-induced switch into mania: a report of two cases. Biol Psychiatry. 2001, 49:468-70. 10.1016/s0006-3223(00)01086-6

22. Critical appraisal tool. (2011). Accessed: April 13, 2021: https://jbi.global/critical-appraisal-tools.

23. Moher D, Liberati A, Tetzlaff J, Altman DG; PRISMA Group: Preferred reporting items for systematic reviews and meta-analyses: the PRISMA statement. PLoS Med. 2009, 6:1000097. 10. 1371/journal.pmed1000097

24. Zhang L, Zhu J, Zhang T, et al.: Comparative efficacy of add-on rTMS in treating the somatic and psychic anxiety symptoms of depression comorbid with anxiety in adolescents, adults, and elderly patients - a realworld clinical application. J Affect Disord. 2020, 276:305-11. 10.1016/j.jad.2020.05.151

25. Croarkin PE, Nakonezny PA, Deng ZD, et al.: High-frequency repetitive TMS for suicidal ideation in adolescents with depression. J Affect Disord. 2018, 239:282-90. 10.1016/j.jad.2018.06.048

26. Sonmez AI, Kucuker MU, Lewis CP, et al.: Improvement in hypersomnia with high frequency repetitive transcranial magnetic stimulation in depressed adolescents: preliminary evidence from an open-label study. Prog Neuropsychopharmacol Biol Psychiatry. 2020, 97:109763. 10.1016/j.pnpbp.2019.109763

27. MacMaster FP, Croarkin PE, Wilkes TC, et al.: Repetitive transcranial magnetic stimulation in youth with treatment resistant major depression. Front Psychiatry. 2019, 10:170. 10.3389/fpsyt.2019.00170

28. Wall CA, Croarkin PE, Maroney-Smith MJ, et al.: Magnetic resonance imaging-guided, open-label, highfrequency repetitive transcranial magnetic stimulation for adolescents with major depressive disorder. J Child Adolesc Psychopharmacol. 2016, 26:582-9. 10.1089/cap.2015.0217

29. Croarkin PE, Nakonezny PA, Wall CA, Murphy LL, Sampson SM, Frye MA, Port JD: Transcranial magnetic stimulation potentiates glutamatergic neurotransmission in depressed adolescents. Psychiatry Res Neuroimaging. 2016, $247: 25-33.10 .1016 /$ j.pscychresns.2015.11.005

30. Wall CA, Croarkin PE, McClintock SM, Murphy LL, Bandel LA, Sim LA, Sampson SM: Neurocognitive effects of repetitive transcranial magnetic stimulation in adolescents with major depressive disorder. Front Psychiatry. 2013, 4:165. 10.3389/fpsyt.2013.00165

31. Wall CA, Croarkin PE, Sim LA, et al.: Adjunctive use of repetitive transcranial magnetic stimulation in depressed adolescents: a prospective, open pilot study. J Clin Psychiatry. 2011, 72:1263-9. 10.4088/JCP.11m07003

32. Bloch Y, Grisaru N, Harel EV, et al.: Repetitive transcranial magnetic stimulation in the treatment of depression in adolescents: an open-label study. J ECT. 2008, 24:156-9. 10.1097/YCT.0b013e318156aa49

33. Cullen KR, Jasberg S, Nelson B, Klimes-Dougan B, Lim KO, Croarkin PE: Seizure induced by deep 
transcranial magnetic stimulation in an adolescent with depression. J Child Adolesc Psychopharmacol. 2016, 26:637-41. 10.1089/cap.2016.0070

34. Cristancho P, Akkineni K, Constantino JN, Carter AR, O'Reardon JP: Transcranial magnetic stimulation in a 15-year-old patient with autism and comorbid depression. J ECT. 2014, 30:e46-7. 10.1097/YCT.0000000000000156

35. Segev A, Spellun J, Bloch Y: Anxiety as a central outcome measure in an adolescent with major depressive disorder treated with repetitive transcranial magnetic stimulation. J ECT. 2014, 30:e54-5. 10.1097/YCT.0000000000000183

36. Chiramberro M, Lindberg N, Isometsä E, Kähkönen S, Appelberg B: Repetitive transcranial magnetic stimulation induced seizures in an adolescent patient with major depression: a case report. Brain Stimul. 2013, 6:830-1. 10.1016/j.brs.2013.02.003

37. Hu SH, Wang SS, Zhang MM, et al.: Repetitive transcranial magnetic stimulation-induced seizure of a patient with adolescent-onset depression: a case report and literature review. J Int Med Res. 2011, 39:203944. 10.1177/147323001103900552

38. Pan F, Li D, Wang X, Lu S, Xu Y, Huang M: Neuronavigation-guided high-dose repetitive transcranial magnetic stimulation for the treatment of depressive adolescents with suicidal ideation: a case series. Neuropsychiatr Dis Treat. 2018, 14:2675-9. 10.2147/NDT.S176125

39. Yang XR, Kirton A, Wilkes TC, et al.: Glutamate alterations associated with transcranial magnetic stimulation in youth depression: a case series. J ECT. 2014, 30:242-7. 10.1097/YCT.0000000000000094

40. Croarkin PE, Wall CA, King JD, Andrew Kozel F, Daskalakis ZJ: Pain during transcranial magnetic stimulation in youth. Innov Clin Neurosci. 2011, 8:18-23.

41. Loo C, Mcfarquhar T, Walter G: Transcranial magnetic stimulation in adolescent depression. Australas Psychiatry. 2006, 14:81-5. 10.1080/j.1440-1665.2006.02251.x

42. Zhang T, Zhu J, Xu L, et al.: Add-on rTMS for the acute treatment of depressive symptoms is probably more effective in adolescents than in adults: evidence from real-world clinical practice. Brain Stimul. 2019, 12:103-9. 10.1016/j.brs.2018.09.007

43. Croarkin PE, Elmaadawi AZ, Aaronson ST, et al.: Left prefrontal transcranial magnetic stimulation for treatment-resistant depression in adolescents: a double-blind, randomized, sham-controlled trial. Neuropsychopharmacology. 2021, 46:462-9. 10.1038/s41386-020-00829-y

44. Walter G, Tormos JM, Israel JA, Pascual-Leone A: Transcranial magnetic stimulation in young persons: a review of known cases. J Child Adolesc Psychopharmacol. 2001, 11:69-75. 10.1089/104454601750143483

45. Magavi LR, Reti IM, Vasa RA: A review of repetitive transcranial magnetic stimulation for adolescents with treatment-resistant depression. Int Rev Psychiatry. 2017, 29:79-88. 10.1080/09540261.2017.1300574

46. Hett D, Rogers J, Humpston C, Marwaha S: Repetitive transcranial magnetic stimulation (rTMS) for the treatment of depression in adolescence: a systematic review. J Affect Disord. 2021, 278:460-9. 10.1016/j.jad.2020.09.058

47. Brim RL, Miller FG: The potential benefit of the placebo effect in sham-controlled trials: implications for risk-benefit assessments and informed consent. J Med Ethics. 2013, 39:703-7. 10.1136/medethics-2012101045

48. Zewdie E, Ciechanski P, Kuo HC, et al.: Safety and tolerability of transcranial magnetic and direct current stimulation in children: prospective single center evidence from 3.5 million stimulations. Brain Stimul. 2020, 13:565-7. 10.1016/j.brs.2019.12.025

49. Krishnan C, Santos L, Peterson MD, Ehinger M: Safety of noninvasive brain stimulation in children and adolescents. Brain Stimul. 2015, 8:76-87. 10.1016/j.brs.2014.10.012

50. OCEBM levels of evidence. (2011). Accessed: June 26, 2020: https://www.cebm.ox.ac.uk/resources/levels-ofevidence/ocebm-levels-of-evidence. 